\title{
A
}

Acta HealthMedica

Acta HealthMedica (ISSN: 2414-6528)

http://www.ActaHealthMedica.com

Volume: 1, Issue: 4, December 2016, Pages: 93-97, DOI: http://dx.doi.org/10.19082/ah93

\section{A RARE CASE OF PRIMARY HYDATIDOSIS; A PRIMARY PARIETAL PLEURAL HYDATID CYST}

Firas Alakhras Aljanadi

M.D., MSc., Damascus University Cardiac Surgery Hospital, Damascus University, Damascus, Syria

\section{TYPE OF ARTICLE: CASE REPORT}

\begin{abstract}
Introduction: Hydatid disease is a parasitic disease that mainly affects the liver and the lungs. Primary parietal pleural hydatid disease is a rare variant of this uncommon disease and is rarely reported in medical literature.

Case Presentation: We report a case of 36-year-old woman presented with chronic cough and chest pain. The blood tests showed high titer of anti-Echinococcus antibodies, and chest x-ray showed a well-defined round homogenous radio-opacity in the right middle and upper zones. A chest computed-tomography scan revealed a cystic lesion in the superior posterior segments of the right lung. However, surgical intervention proved it is a hydatid cyst arising from the parietal pleura; it was removed completely, and the patient improved significantly.

Conclusion: Primary pleural hydatid disease is rare. Though it is rare, pleural hydatid disease should be kept in mind, especially in the deferential diagnosis of a mass lesion in the chest. In addition to that, the appropriate surgical precautions should be taken and the en-bloc complete resection of the cyst may prevent recurrence and spreading of hydatid disease.

KEYWORDS: Hydatid cyst; Echinococcus granulosus; Pulmonary; Parietal pleura
\end{abstract}

\section{INTRODUCTION}

Hydatid disease is a parasitic zoonosis caused by the larval stage of the tapeworm Echinococcus granulosus. Humans can be infected when they pick up worm eggs from contaminated dogs during their childhood or contaminated uncooked vegetables in sheep-raising areas of the world (1). It is still an endemic disease in some of the following regions: South America, North Africa, Asia, and Australia (1-2). Liver cysts are the most common form of human Echinococcosis (2). While the lungs are the second most common site of lodgment, extrapulmonary thoracic hydatidosis are rare (2). The extrapulmonary hydatid cyst on the pleura as a primary site is a rare variant of this uncommon disease (2). In this case, we aim to report a rare type of hydatid cyst disease arising solely from the parietal pleura. Without pulmonary or hepatic involvement, this has rarely been previously reported. It presented resembling an intrathoracic mass, causing chest pain and recurrent chest infections. The precise site was confirmed by surgery, which revealed a hydatid cyst on the parietal pleura on the interior surface of the chest wall. In addition to that, we aim to report how we approached this untypical case.

\section{CASE PRESENTATION}

\subsection{Patient's Characteristics}

A 36-year-old woman presented to the hospital with a three-month history of chronic cough and chest pain. The cough is mostly dry with frequent attacks of morning productive cough with non-bloody sputum and without a specific smell, associated with a dull right chest pain under the breast with episodic sharp pain lasting for few minutes before it resolves spontaneously. She also complained of exertional dyspnea NYHA II. These symptoms relieve slightly with medical therapy (painkiller and bronchodilators). There was no fever or hemoptysis. Previously, she had pneumonia five years ago. She was a housewife living in a sheep-raising area.

\subsection{Clinical Findings}

On physical examination, the chest was moving symmetrically; there was no hyper resonance or dullness, and air entry was bilaterally equal.

\section{Correspondence:}

Dr. Firas Alakhras Aljanadi, Damascus University Cardiac Surgery Hospital, Damascus University, Damascus, Syria. Tel: +44.7405163591, Email: firasaljanadi@gmail.com

Received: November 8, 2016, Accepted: November 21, 2016, Published: December 2016

iThenticate screening: November 9, 2016, English editing: November 22, 2016, Quality control: November 29, 2016

(C) 2016 The Authors. This is an open access article under the terms of the Creative Commons Attribution-NonCommercialNoDerivs License, which permits use and distribution in any medium, provided the original work is properly cited, the use is non-commercial and no modifications or adaptations are made. 


\subsection{Diagnostic Focus and Assessment}

The electrocardiography (ECG) was normal, and the blood tests also were normal except for both the erythrocyte sedimentation rate (ESR), which was $42 \mathrm{~mm} / \mathrm{hr}$ (normal range $0-29 \mathrm{~mm} / \mathrm{hr}$ ), as well as the Echinococcus granulosus antibodies test, which was significantly high 1:800 (1:160 and higher dilutions was considered as positive). The tuberculin skin test was positive with $10 \mathrm{~mm}$ of induration after 72 hours; however, sputum culture for tuberculosis(TB) was negative. The chest $\mathrm{x}$-ray (Figure 1) showed an almost well-defined round homogenous radioopacity in the right middle and upper zones. Differential diagnoses on a chest x-ray included fluid-filled cysts, benign tumors, carcinoma, metastases, and inflammatory masses. The chest contrast-enhanced computedtomography images (Figures 2,3) revealed a well-defined round lobulated cystic lesion with noticeable presence of mildly thickened wall with contrast enhancement (ring enhancement sign) containing fluid, located in the superior and posterior segments of the right lung, measures $65 \mathrm{~mm}$ × $35 \mathrm{~mm}$ with a deferential diagnoses of either a pulmonary abscess or a complicated hydatid cyst.

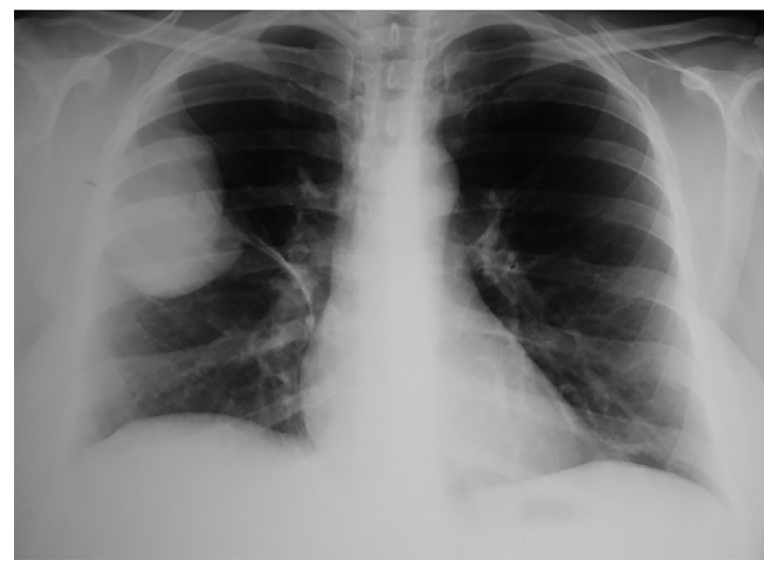

Figure 1. Chest $x$-ray: A posterior anterior view of chest $x$-ray showing almost well-defined round dense homogenous radio-opacity in right middle and upper zones.
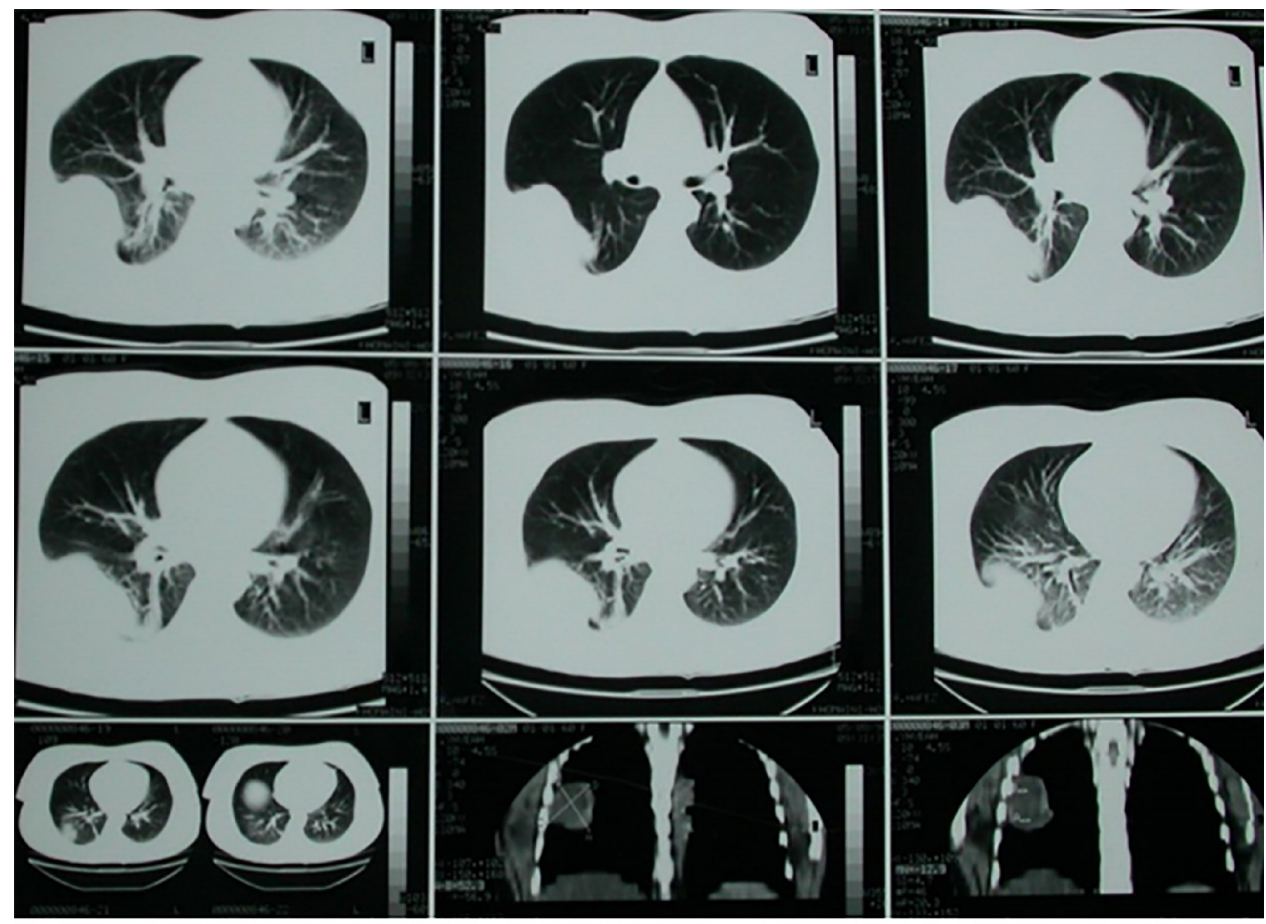

Figure 2. Chest computed-tomography images (axial view with pulmonary window and reformatted coronal reconstruction): Axial images show a well-defined round lobulated cystic lesion, while coronal reconstruction views show the cystic lesion had a thickened wall with contrast enhancement (ring enhancement sign), located in the right superior and posterior segments, measures $65 \mathrm{~mm}$ x $35 \mathrm{~mm}$. 


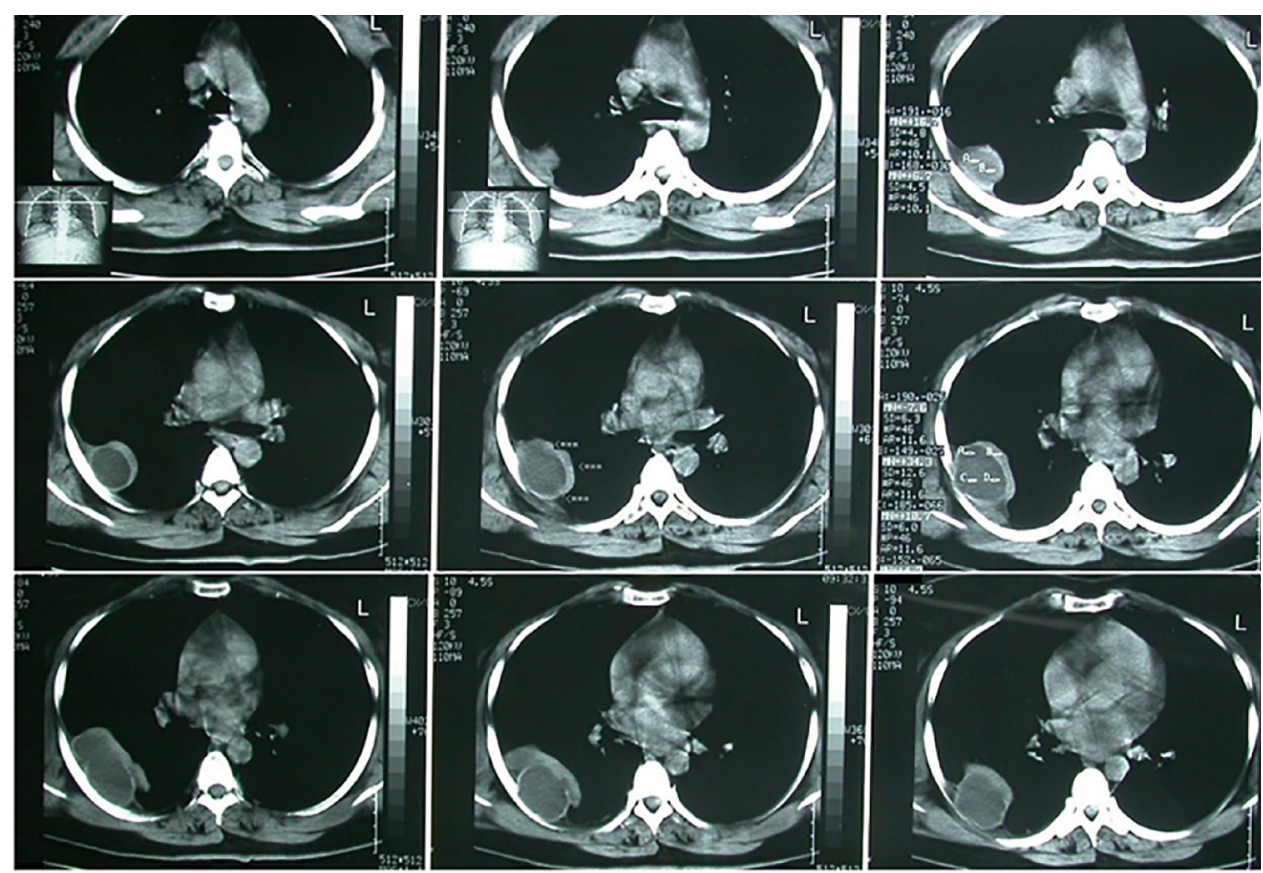

Figure 3. Chest computed-tomography images (mediastinal window): Axial contrast-enhanced computedtomography images show a well-marginated round lobulated cystic mass with homogeneous attenuation, which showed a thickened wall with contrast enhancement (ring enhancement sign), and located in the superior and posterior segments of the right lung.

\subsection{Therapeutic Focus and Assessment}

Taking into consideration that it was most probably a hydatid cyst, the surgical management was preceded with right posterolateral thoracotomy; to prevent spillage of the cyst content, we flooded the surgical field with pads soaked in hypertonic $20 \%$ sodium chloride solution. We found a hydatid cyst arising from the parietal pleura protruding in the pleural cavity with significant adhesions to the right lung and the chest wall. To inactivate daughter cysts and scolices, prior removal we injected $20 \%$ hypertonic saline solution into the cyst at its most superficial point; five minutes later it was aspirated completely and directly toward the aspirator. After that, it was mobilized, dissected, and removed completely with its membranes en-bloc. The adhesions were freed totally, and the pleural cavity was washed with the hypertonic solution and a povidone-iodine solution. Afterward, we inflated the lung to check for air leaks and closed any bronchial openings with a 2-0 nonabsorbable suture material. A haemostasis for bleeding from the chest wall was followed, and no reconstruction was needed. Two drains were left in the pleural cavity, and the thoracotomy was closed after that.

\subsection{Follow-up and Outcomes}

There was complete cyst removal in our case. The post-operative period was uneventful (Figure 4; the fourth postoperative day chest X-ray). The patient improved markedly and was discharged on Albendazol (10mg/kg) for three months. After 10 months of surgery, the patient has no evidence of recurrence and is completely disease and symptom free (Figure 5). 


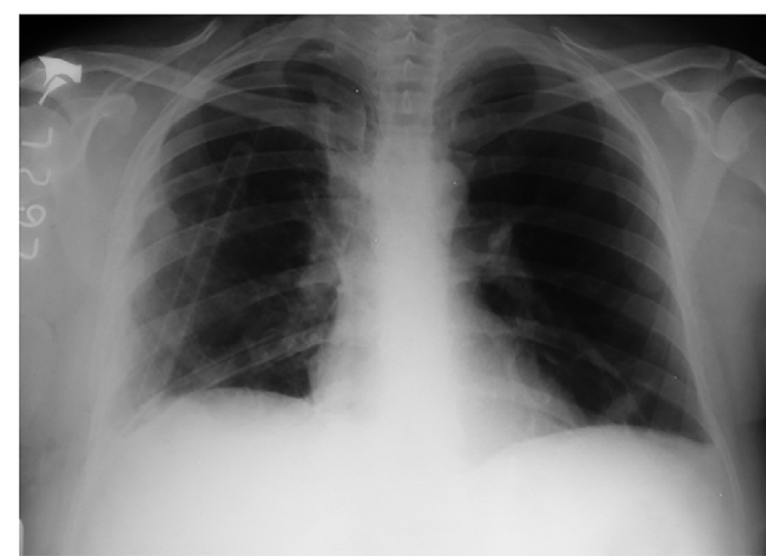

Figures 4. The fourth post-operative day chest $\mathrm{x}$-ray.

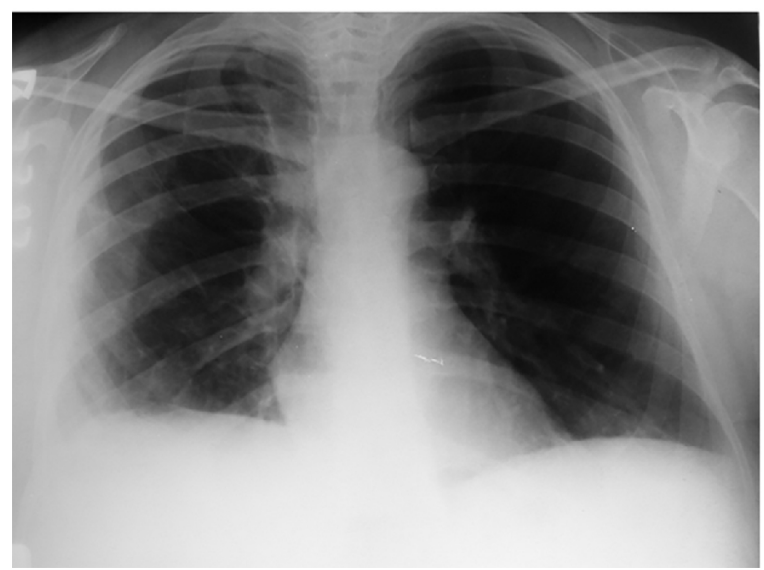

Figures 5. Follow-up chest x-ray.

\section{DISCUSSION}

Primary hydatid cysts rarely form in intrathoracic yet extrapulmonary sites (3). Generally, hydatid disease results from hematogenic invasion of the liver (50\%-60\%) and the lung (10\%-30\%) by Echinococcus granulosus and intrathoracic extrapulmonary localization of thoracic cysts is observed in $7.4 \%$ of cases. Among intrathoracic extrapulmonary hydatid cysts, $55 \%$ of them are localized in the fissure, $18 \%$ within the parietal ple ura, $14 \%$ in the chest wall, $4.5 \%$ in the mediastinum, and $4.5 \%$ in the diaphragm (3). According to that (3), parietal pleura is affected in $1.3 \%$ of all cases. Almost always it is secondary while primary cases are rare. In other words, rupture of a pulmonary hydatid cyst into the pleural space, either spontaneously or during surgery, is the most common cause of pleural hydatidosis or chest wall hydatidosis; however, primary pleural hydatid disease is very rare (4). Previously reported pleural cysts have developed chiefly as a result of perforation of the cysts into the pleural area and by diaphragmatic transmission (5). This is not actually pleural echinococcosis but a complication thereof. The real clinical feature as seen in our patient, the cyst was arising solely from the parietal pleura primarily in the pleural layers and the pleural region. In this current case, the possible mechanism of primary hydatid disease of the parietal pleura may be as follows: the embryo passes through the duodenal wall into either the portal vein or the Periduodenal and perigastric lymphatics. Periduodenal and perigastric lymphatic channels connect with the thoracomediastinal lymphatic and the thoracic duct (6). This mechanism may explain the development of primary chest wall hydatid disease in the absence of pulmonary or hepatic cysts (7). Although hydatid cysts usually produce various symptoms, they can be asymptomatic. Intrathoracic extrapulmonary cysts may produce compression symptoms in surrounding vital structures (5). Our patient chiefly experienced chronic cough, pleuritic chest pain, and dyspnea consequent to lung compression and irritation of parietal pleura. Because primary pleural hydatid cysts is rare, it can create difficulties in diagnosis or reaching an accurate preoperative diagnosis, which might be followed by unexpectedly necessary wide resections and reconstructive procedures. In the case of intrathoracic extrapulmonary cysts, preoperative diagnostic methods are not always reliable (5). Difficulties in diagnosis may lead to an incorrectly conceived initial surgical approach (5). As in our case, where the chest x-ray and CT scan were 
confusing referring to a pulmonary cystic lesion which could affect the planned approach and surgical entry site. Therefore, Echinococcus antibodies test is advised whenever there is a mass on the chest radiographs, especially in the endemic areas, and it is important that required surgical precautions be taken in every case where hydatid cyst is among the differentials.

Corrective surgical procedures necessarily differ from those that are used to treat the far more typical pulmonary or hepatic hydatid cysts (5). In addition to that, approaches to extrapulmonary hydatid cysts that are too conservative may lead to spillage and recurrence, so en-bloc excision is the recommended technique to prevent recurrence. Treatment of choice for hydatid cyst is surgical removal. The precise location of an intrathoracic extrapulmonary cyst is usually confirmed during surgical intervention as in our case and the corrective surgical procedures, were not required. As a general rule, when the presurgical diagnosis of hydatid cyst is suspected, surgeon should take care of four things in order to achieve complete resection and to avoid recurrence of disease from pleural hydatid cysts: 1) Planning the appropriate surgical approach to prevent cystic rupture or spillage when doing the thoracotomy; 2) inactivation of daughter cysts and scolices prior to removal by injecting $20 \%$ hypertonic saline solutions into the cyst; 3) no spillage of cyst contents during surgery to avoid anaphylactoid reaction, recurrence, and multiple hydatidosis; 4) complete removal of the cyst including the innermost germinative layer, which can produce scolices, with en-bloc excision whenever possible; sometimes, to avoid recurrence, it is necessary to resect the affected surrounding tissues completely; postoperatively, it may be necessary to place patients on an anthelmintic medical regimen (Albendazol) with appropriate follow-up reevaluations.

\section{CONCLUSIONS}

Hydatid cysts can form in different anatomic sites, but primary pleural hydatid disease is very rare. This rarity may cause difficulties in diagnosis. However, pleural hydatid disease should be kept in mind, especially in the deferential diagnosis of a mass lesion in the chest. In addition to that, the appropriate surgical precautions that should be taken, and the en-bloc complete resection of the cyst may prevent recurrence and spreading of hydatid disease.

\section{ACKNOWLEDGMENTS:}

I would like to thank the Thoracic Surgery Department and the surgical, anaesthetic, and theatre staff at AlMouwasat University Hospital for their cooperation and forbearance.

\section{CONFLICT OF INTEREST:}

There is no conflict of interest to be declared.

\section{REFERENCES:}

1) Burgos L, Baquerizo A, Munoz W, de Aretxabala X, Solar C, Fonseca L. Experience in the surgical treatment of 331 patients with pulmonary hydatidosis. J Thorac Cardiovasc Surg. 1991; 102: 427-30 .PMid: 1881181.

2) Srinivasan B, Mohite PN, Thingnam SK. Extrapulmonary intrapleural hydatid cysts -rare variant of uncommon disease. Indian J Thorac Cardiovasc Surg. 2010; 26: 24750. doi: 10.1007/s12055-010-0054497.

3) Oguzkaya F, Akcali Y, Kahraman C, Emirogullari N, Bilgin M, Sahin A. Unusually located hydatid cysts: intrathoracic but extrapulmonary. Ann Thorac Surg 1997; 64(2): 334-7. doi: 10.1016/S00034975(97)00521-3.

4) Özdemir N, Akal M, Kutlay H, Yavuzer S. Chest wall echinococcosis. Chest. 1994; 105: 1277-9. PMid: 8162770

5) Gürsoy S., Ucvet A., Tozum H., Erbaycu A. E., Kul C., Basok O. Primary intrathoracic extrapulmonary hydatid cysts. Texas Heart Institute Journal. 2009; 36(3): 230-233. PMid: 19568393.

6) Sarsam A. Surgery of pulmonary hydatid cysts. Review of 155 cases. J Thorac Cardiovasc Surg. 1971; 62(4): 663-8. PMid: 5093823.

7) Findikcioglu A, Kilic D, Canpolat T, Hatipoglu A. Primary hydatid disease of the chest wall. Ann Thorac Cardiovasc Surg. 2007; 13(3): 203-5. PMid: 17592431. 